\title{
Microglial replacement therapy: a potential therapeutic strategy for incurable CSF1R-related leukoencephalopathy
}

Jinming Han ${ }^{*^{*} \mathbb{D}}$, Heela Sarlus ${ }^{1}$, Zbigniew K. Wszolek ${ }^{2}$, Virginija Danylaité Karrenbauer ${ }^{3,4^{*}}$ and Robert A. Harris ${ }^{1}$

\begin{abstract}
CSF1R-related leukoencephalopathy is an adult-onset leukoencephalopathy with axonal spheroids and pigmented glia caused by colony stimulating factor 1 receptor (CSF1R) gene mutations. The disease has a global distribution and currently has no cure. Individuals with CSF1R-related leukoencephalopathy variably present clinical symptoms including cognitive impairment, progressive neuropsychiatric and motor symptoms. CSF1R is predominantly expressed on microglia within the central nervous system (CNS), and thus CSF1R-related leukoencephalopathy is now classified as a CNS primary microgliopathy. This urgent unmet medical need could potentially be addressed by using microglia-based immunotherapies. With the rapid recent progress in the experimental microglial research field, the replacement of an empty microglial niche following microglial depletion through either conditional genetic approaches or pharmacological therapies (CSF1R inhibitors) is being studied. Furthermore, hematopoietic stem cell transplantation offers an emerging means of exchanging dysfunctional microglia with the aim of reducing brain lesions, relieving clinical symptoms and prolonging the life of patients with CSF1R-related leukoencephalopathy. This review article introduces recent advances in microglial biology and CSFIR-related leukoencephalopathy. Potential therapeutic strategies by replacing microglia in order to improve the quality of life of CSF1R-related leukoencephalopathy patients will be presented.
\end{abstract}

Keywords: CSF1R-related leukoencephalopathy, Microglia, Microglial replacement

\section{Introduction}

CSF1R-related leukoencephalopathy, a subgroup of adult-onset leukodystrophy, is a progressive neurodegenerative white matter disease caused by mutations in the colony stimulating factor 1 receptor (CSF1R) gene [1]. CSF1R is highly expressed on microglia within the central nervous system (CNS) and thus CSF1R-related leukoencephalopathy is considered to be a primary CNS

\footnotetext{
*Correspondence: jinming.han@ki.se; virginija.karrenbauer@ki.se ${ }^{1}$ Applied Immunology and Immunotherapy, Department of Clinical Neuroscience, Karolinska Institutet, Center for Molecular Medicine, Karolinska University Hospital, Solna, Sweden

${ }^{3}$ Department of Clinical Neuroscience, Karolinska Institutet, Stockholm, Sweden

Full list of author information is available at the end of the article Virginija Danylaité Karrenbauer and Robert A. Harris shares senior authorship.
}

microgliopathy. The prognosis is extremely poor [2]. The terms of pigmented orthochromatic leukodystrophy (POLD) and hereditary diffuse leukoencephalopathy with spheroids (HDLS) were previously used to describe the disease spectrum [3, 4]. Specifically, HDLS was first reported in a Swedish family as early as 1984 based on the pathological hallmarks, including widespread white matter degeneration with numerous neuroaxonal spheroids, and the accumulation of lipid-laden and pigmented macrophages [5]. However, the alanyl tRNA synthetase $(A A R S)$ gene mutation has recently been identified in the original Swedish HDLS family, who have also been tested for CSF1R gene mutation status with negative results [6]. However, all POLD families tested for CSF1R gene mutations have been positive for the mutation [7]. To avoid further nomenclature confusion and adopting current nomenclature trends, we will use the term of original author(s) and the source, provide a link to the Creative Commons licence, and indicate if changes were made. The images or other third party material in this article are included in the article's Creative Commons licence, unless indicated otherwise in a credit line to the material. If material is not included in the article's Creative Commons licence and your intended use is not permitted by statutory regulation or exceeds the permitted use, you will need to obtain permission directly from the copyright holder. To view a copy of this licence, visit http://creativecommons.org/licenses/by/4.0/. The Creative Commons Public Domain Dedication waiver (http://creativeco mmons.org/publicdomain/zero/1.0/) applies to the data made available in this article, unless otherwise stated in a credit line to the data. 
CSF1R-related leukoencephalopathy, rather than HDLS or POLD, in this review.

Clinically, patients with CSF1R-related leukoencephalopathy typically manifest progressive cognitive decline, behavioral or personality changes and motor signs reminiscent of atypical Parkinsonism. CSF1R-related leukoencephalopathy may be misdiagnosed as frontotemporal dementia or dementia with Lewy bodies, cerebral autosomal dominant arteriopathy with subcortical infarcts and leukoencephalopathy (CADASIL), or primary progressive multiple sclerosis (MS), due to the overlapping clinical features and magnetic resonance imaging (MRI) abnormalities [8-10]. The age of disease onset in patients with CSF1R-related leukoencephalopathy varies from 10 to 78 years old with an average age of 43 years $[4,11]$. The average survival is reported from 5.3 to 6.8 years $[8,12]$ but with rather wide range from 2 to 34 years [13]. Hyperintense signals of subcortical white matter in CSF1R-related leukoencephalopathy can rarely be extended to the cervical and thoracic spinal cord areas [14]. Optic nerve damage and myelitis may also occur in patients with CSF1R-related leukoencephalopathy, which confounds the diagnosis $[9,15]$. Detailed clinical and radiological examinations are therefore of diagnostic importance. Konno and colleagues recently proposed the diagnostic criteria for CSF1R-related leukoencephalopathy, providing 'probable' or 'possible' designations for patients without a genetic test, and yielding high sensitivity and specificity for diagnosis [3]. Both genetic and pathological examinations are required for a confirmed diagnosis. It is important to note that there is no currently available, internationally used standardized and validated $C S F 1 R$-related leukoencephalopathy functional score scale. It is thus challenging to compare the evaluation of disease phenotypes and to evaluate experimental treatment clinical or surrogate endpoints due to the multifaceted clinical phenotypes.

A diagnostic value of unique calcifications distributed in selective brain regions on computed tomography scans has been proposed [16]. In addition, thinning of the corpus callosum, extensive non-confluent and occasionally even confluent subcortical white matter lesions on T2-weighted brain MRI images, and persistent restricted diffusion of diffuse-weighted imaging signal without gadolinium enhancement can be evident during the disease course. This may help in identifying potential CSF1R-related leukoencephalopathy cases (Fig. 1) [17]. We have previously demonstrated that the brain volume fractions measured by MRI did not differ significantly between CSF1R-related leukoencephalopathy and MS patients, while the cerebellum was relatively spared in CSF1R-related leukoencephalopathy patients [18]. We also employed an artificial intelligence machine learning to differentiate this disease state from MS using multiparametric brain MRI images [19].

As there are no laboratory findings specific for CSF1Rrelated leukoencephalopathy, detailed clinical examinations are important to exclude differential diagnoses. Strikingly, neurofilament light chain, a marker of axonal injury [20], can serve as a potential biomarker for CSF1Rrelated leukoencephalopathy as higher levels are evident in both the serum and cerebrospinal fluid of these patients compared to in MS patients [21]. The level of neurofilament light chain already starts to increase in young CSF1R mutation carriers who exhibit no obvious clinical symptoms [21]. These features may assist in improving the diagnostic process and in differentiating CSF1R-related leukoencephalopathy white matter changes from other conditions. More importantly, it can serve as a potential biomarker for monitoring the response to therapeutic approaches.

The prevalence of CSF1R-related leukoencephalopathy is probably underestimated, but is gradually increasing mainly due to more widespread use of novel genetic tests such as next-generation sequencing [22]. As a genetic disease, CSF1R-related leukoencephalopathy causes emotional distress for patients and their families and poses a global burden for society. Patients can only be supported through maintaining nutrition and physical rehabilitation programs. Since microglia, the CNS-resident immune cells, are affected in this condition, developing immune cell-based therapies in order to improve disease prognosis and the quality of life of patients with CSF1R-related leukoencephalopathy represents a potential strategy to meet this urgent medical need.

\section{The role of CSF1R in CSF1R-related leukoencephalopathy and related mutant rodent models}

The CSF1R cell-surface receptor is mainly expressed on microglia within the CNS [23], and as previously reviewed, the survival, maintenance and proliferation of microglia is critically dependent on CSF1R [24, 25]. Over 70 distinct mutations in the gene have been identified [2], most being located at the tyrosine kinase domain of CSF1R on chromosome 5 and encoded by exons 12-21 [4]. CSF1R-related leukoencephalopathy is supposed to be caused by the haploinsufficiency of csflr gene [26]. Homozygous mutations of the CSF1R gene have recently been reported to cause a complete loss of microglia in the brain of an infant who died at 10 months of age [27].

CSF1R mutations cause a decreased activation of JNK, one of the downstream kinases involved in CSF1R signaling [28]. Expression of the CSF1R signaling ligands CSF-1 (predominant in the cerebellum) and interleukin (IL)-34 (predominant in the cortex) are important for microglial homeostasis in both mice and humans, 


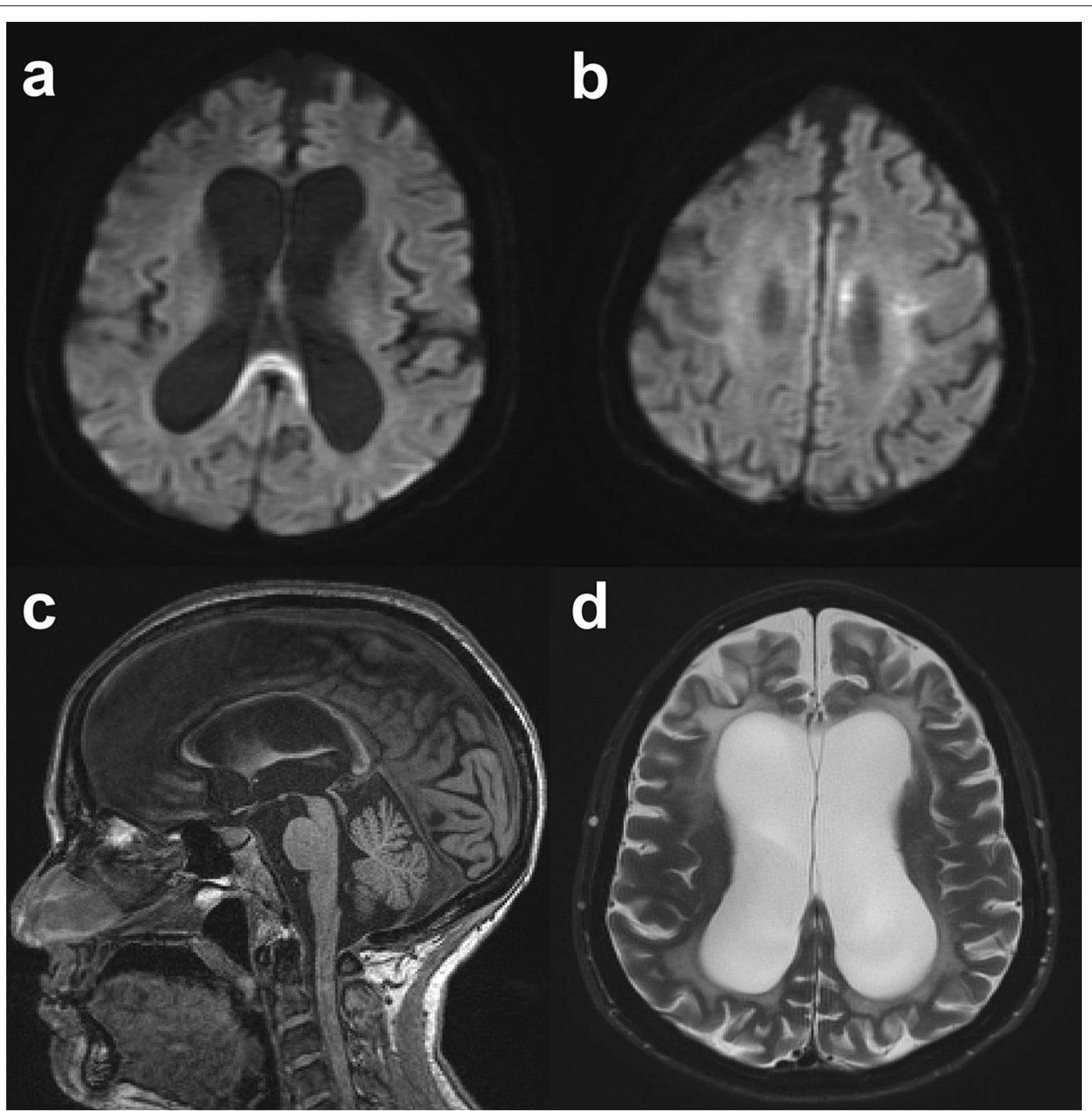

Fig. 1 Brain MRI features of CSF1R-related leukoencephalopathy. The images were obtained from a 47-year-old female with progressive cognitive and behavioral deficits, and motor impairments with an approximate 3-year disease duration. Her father died of progressive dementia. The commercial genetic testing demonstrated the presence of heterozygote CSF1R mutation, p.L786S, c.2357 T>C. a, b Restricted diffusion in the splenium and left body of corpus callosum. c Thinning of corpus callosum on the sagittal section. $\mathbf{d}$ T2 hyperintensities in frontal and parietal white matter on the axial section. Courtesy of Daniel F. Broderick, M.D., Mayo Clinic Florida

indicating brain region-specific effects [29]. It is acknowledged that CSF1R-related leukoencephalopathy is caused by dysfunctional microglia, and that different mutation locations of the CSF1R gene lead to different clinical phenotypes of CSF1R-related leukoencephalopathy, with both disease presentation and progression varying significantly among family members [30, 31]. Although CSF1R-related leukoencephalopathy is a genetic disease inherited in an autosomal dominant pattern, approximately $36 \%$ of cases are sporadic [11]. This can be partly explained by the formation of mosaicism occurred at any cell division, allowing for survival and causing discrepancies of clinical phenotypes [32]. Other environmental factors may also contribute to sporadic cases.

As CSF1R is also expressed on other myeloid cells, CSF1R mutations may thus also affect myeloid cell function in the periphery and contribute to the pathogenesis [33-35]. Several novel observations support this idea. A defined CSF1R variant in one CSF1Rrelated leukoencephalopathy patient was associated with a progressive reduction in the frequency of circulating non-classical monocytes during disease course, since non-classical monocytes express the highest CSF1R levels [33]. Whether circulating non-classical 
monocytes infiltrate into the CNS or that they lose the ability to survive and proliferate in the circulation during disease progression remains unclear.

Microglia appear to be highly sensitive to the effects of CSF1R mutations [36]. While other tissue macrophages such as Langerhans cells in the skin and Kupffer cells in the liver also depend on CSF1R signaling [36], the effects of CSF1R mutations on these tissue macrophages in CSF1R-related leukoencephalopathy patients remains unknown. If additional factors or circulating monocytes may compensate for the loss of these tissue macrophages in CSF1R-related leukoencephalopathy requires further investigation.

Homozygous mutations of the CSF1R gene cause a generalized increase in bone density and metaphyseal dysplasia by influencing osteoclasts, suggesting that CSF1R deficiency may have an additional role in skeletal abnormalities [27]. CSF1R can also be expressed on some neurons and neuronal stem cells [2,37]. Cux1 is a transcriptional factor associated with axonal projection and the numbers of $\mathrm{Cux}^{+}$neurons are reduced in $C S F 1 R$-related leukoencephalopathy patients compared to age-matched controls [27]. Potential effects of CSF1R mutations on these components, including impaired communication between microglia and neurons, warrant further investigation.

Rodent models of CSF1R-related leukoencephalopathy based on Csf1r mutations have been developed and exhibit some of the human disease features. The brain abnormalities of $\mathrm{Csflr}^{-1-}$ mice are typically characterized by significant microglial loss and enlarged ventricles, but these mice die at a very young age. Conversely, Csfl $1 r^{+/-}$mice can survive during adulthood period but then develop abnormal symptoms including cognitive decline and depression with anxiety-like behavior [28]. Skeletal abnormalities are evident in $C s f 1 r^{-1-}$ mice but not in $C s f 1 r^{+/-}$mice [28]. Unlike Csf1r ${ }^{-1-}$ mice, most $C s f 1 r^{-1-}$ rats can survive into adulthood [38]. Despite $\mathrm{Iba}^{+}$microglia being absent in the brain of Csf1r ${ }^{-/-}$ rats there are few apparent structural brain abnormalities at 3 months of age. In addition, $C s f 1 r^{-1-}$ rats exhibit postnatal growth retardation, skeletal abnormalities and infertility [38]. Furthermore, SIRPa ${ }^{+}$circulating monocytes and $\mathrm{CD} 68^{+}$liver Kupffer cells are less affected in heterozygotic rats, but the numbers of these immune cells are significantly reduced in $C s f 1 r^{-/-}$ homozygotic rats [38]. Overall, Csf1r-deficient rodent models on specific genetic backgrounds provide useful information for exploring the pathophysiology of CSF1R-related leukoencephalopathy and potential treatments.

\section{Pathological features of CSF1R-related leukoencephalopathy}

Neuropathological features of CSF1R-related leukoencephalopathy include a widespread loss of myelin and axons, astrogliosis and macrophage accumulation in the presence of swollen and spherical axons [31]. Microglia are adapted to the CNS microenvironment and express specific gene signatures (P2ry12, Tmem119 and SiglecH) in order to perform tissue-specific functions [39]. In CSF1R-related leukoencephalopathy, loss of Iba $1^{+} / \mathrm{P} \mathrm{ry} 12^{+}$microglia were observed in vulnerable cerebral white matter, but not in unaffected grey matter [40]. Microglia exhibit a distinct morphology with narrow cytoplasm and fragmented processes containing beaded structures, differing from activated microglia that are characterized with retracted processes and enlarged cytoplasm in e.g. Alzheimer's disease (AD) [41]. Microglial distribution has been suggested to be regulated by CSF1R [42], and the uneven distribution of microglia in the CSF1R-related leukoencephalopathy brain supports this notion [41]. While quantification of microglia in CSF1R-related leukoencephalopathy tissues is challenging, both immunohistochemical and western blot analyses indicate a reduction in microglial immunoreactivity in patient's brains compared to in controls [41], despite a high proportion of microglia undergoing proliferation and particularly in areas with dense microglial occupation [41]. This suggests that microglia in CSF1R-related leukoencephalopathy retain the ability to proliferate, and that the reduction in their numbers might actually reflect reduced survival, given that CSF1R is important for microglial survival [43].

Infiltrating $\mathrm{Iba}^{+} \mathrm{P} 2 \mathrm{ry} 12^{-}$macrophages are detectable on histopathological examinations [4, 41], particularly in the degenerating corpus callosum, and most express CD68, CD163 and CD204 [41]. There is a dominance of $\mathrm{CD}_{163}{ }^{+}$over $\mathrm{CD}_{68}{ }^{+}$subpopulations present in the pigmented cells [31]. Interestingly, macrophages are evident in areas with sparsely distributed microglia, suggesting that macrophages are recruited to the degenerated white matter and might contribute to pathology. We have previously reported that long-term microglial depletion leads to monocyte colonization of the empty niche [44]. The functional role of infiltrating monocyte-derived cells in CSF1R-related leukoencephalopathy remains to be investigated [40].

\section{Sex differences in CSF1R-related leukoencephalopathy: do microglia play a role?}

Although there is no sex difference in the prevalence of CSF1R-related leukoencephalopathy, it was reported that female patients develop clinical symptoms significantly earlier than do men $[8,12]$. This interesting finding was 
further supported in a Chinese cohort containing 15 patients from 10 unrelated families, with younger age of disease onset in female than in male patients (34.2 vs. 39.2 years) [45]. Motor dysfunction was the most common initial symptom in women. Human sex hormones can mediate potential sex differences of microglia with exogenous estradiol enhancing humoral immunity while testosterone has an opposite effect [46]. The importance of sex-related differences in CSF1R-related leukoencephalopathy disease course requires further investigation [8].

Experimental studies have convincingly demonstrated sex-dependent structural and functional differences in microglia, which is drastically changing our viewpoint $[47,48]$. Iba $1^{+}$microglial cell density in the cortex, hippocampus and amygdala is higher in adult male mice than in females [47]. Microglia in male mice express more MHC-related genes compared to females, suggesting that the antigen-presenting potential of microglia is distinct between males and females in mice [47]. Obvious transcriptional and translational properties of microglia were also noted between male and female mice [47].

Sex differences are more prominent in the settings of age-related disorders since microglial functionality becomes sexually divergent with aging [49]. Unlike in aged male mice, the expression of proinflammatory cytokines in the brain was increased in aged female mice in response to peripheral lipopolysaccharide (LPS) stimulation [50]. Brain-derived neurotrophic factor (BDNF) is produced by microglia and partial ablation of BDNF had different effects on proinflammatory cytokine production in the brains of male mice compared to females in response to LPS. This suggests that BDNF plays a role in mediating sex-dependent difference in intrathecal inflammatory responses to LPS [51].

Sex differences of microglial functions can be maintained even after they are transferred into the brain, as illustrated by transplantation of female microglia into the male brain, which conferred protection against stroke [48]. The impact of the microbiome on microglial properties is also sex-dependent, the long-term absence of the microbiome exhibiting more profound changes in females during adulthood [52]. Recently, we also observed sex-dependent severity of experimental autoimmune encephalomyelitis in adult mice following engraftment of microglia-like cells [53].

Although well documented, there are no consistent data proving sex-dependent effects during neurological diseases. However, these available preclinical results set the basis for further studies to explore how sex differences in human microglia might contribute to sex dependent phenotypic differences in CSF1R-related leukoencephalopathy patients. This may also provide translational evidence for developing potential microglial sex-specific therapies for CSF1R-related leukoencephalopathy.

\section{The microglial niche theory: a developing story}

The macrophage niche theory, a novel concept first described in the macrophage research field, postulates that each macrophage cell occupies its own territory [54, 55 ] and that competitive repopulation of an empty macrophage niche is tightly sensed and regulated [44]. The depletion or death of tissue resident macrophages may provide an empty space, which triggers the proliferation of neighboring macrophages to repopulate the empty niche [55].

All tissue resident macrophage populations are established during development by embryonic precursors derived from either the yolk sac or from fetal liver monocytes [56]. In some tissues such as the brain, the embryonically derived macrophages maintain themselves during adulthood by local self-renewal [57], whereas in tissues such as the dermis, gut and pancreas the macrophages are maintained by continual replacement from the blood-circulating monocyte pool $[58,59]$. Embryonic and adult macrophage precursors have similar abilities to differentiate into macrophages in diverse tissues, where each tissue accommodates a limited number of macrophage niches and the process of macrophage differentiation only halts once the niche is fully occupied [54].

Microglia in the CNS are unique in that the microglial precursors derived from immature erythromyeloid progenitors populate the CNS around embryonic day 8.0, before formation of the blood-brain barrier, and they differentiate locally into microglia [60]. Subsequent closure of the blood-brain barrier prevents further colonization of the CNS with additional pools of precursors. During homeostasis microglia remain separated from the circulation and maintain their numbers through local proliferation without replenishment by blood monocytes $[57,60]$. Additional niches can be formed during organ growth during the neonatal period [54]. Microglial niches can become temporarily available under specific circumstances, such as following irradiation-mediated damage that can cause the leakiness of barriers and subsequent recruitment of immune cells from the peripheral pool $[54,61]$. Furthermore, depletion using a selective inhibitor of CSF1R provides an almost empty microglia niche without disrupting the blood brain barrier [43]. The few remaining microglia repopulate the CNS through hyperproliferation and exceed the original numbers of depleted microglia, which eventually normalizes to the baseline level [62, 63]. Depending on experimental depletion approaches, the newly repopulated microglia can either arise from remaining resident microglia [64] or infiltrating microglia-like cells [44, 65-67]. 
Kupffer cells enhance their proliferative ability to repopulate the niche when they are partly ablated [58]. A higher proliferative rate of microglia in selective brain regions has also been noted in CSF1R-related leukoencephalopathy patients through pathological examinations [41]. However, the numbers of microglia are significantly decreased in some but not all brain regions in patients, suggesting that signals involved in repopulating the microglial niche may be damaged in selective brain regions during CSF1R-related leukoencephalopathy [41]. Artificial administration of neurotrophic factors such as BDNF may also be necessary when the microglial niche is made available during CSF1R-related leukoencephalopathy $[68,69]$. A better understanding of specific microglial niches and their associated regulatory molecular pathways is thus necessary in order to effectively design microglial replacement therapies for CSF1R-related leukoencephalopathy patients.

\section{Microglial replacement by the proliferation of resident microglia}

The mechanisms of cell death including apoptosis, necroptosis and pyroptosis provide an avenue to stimulate the replacement of accumulated and unwanted cells with new cells in order to maintain proper tissue functions [70]. Specifically, microglia are self-maintained in the CNS through a balance between proliferation and apoptosis with little contribution from peripheral cells under physiological conditions [57]. Microglial depletion through conditional genetic depletion or pharmacological therapies is now considered as effective approach to permit new microglia to repopulate the CNS niche [25, 44]. By doing so, subsequent microglia replenishment might have the potential to compensate for the cell loss and resolve ongoing neuroinflammation [71].

Pharmacological targeting of microglia using CSF1R inhibitors such as PLX3397 [72], PLX5622 [73], BLZ945 [74], GW2850 [75], Ki20227 [76] and JNI-40346527 [77] are increasingly used to deplete microglia. These drugs can be integrated into rodent chow diets and eliminate microglia in the CNS by crossing the blood brain barrier without affecting the health and growth of adult mice $[24,78]$. It was suggested that newly repopulating microglia following such depletion stemmed from the Nestin $^{+}$neuro-progenitors [43], while this was disputed in a later fate mapping study which indicated that repopulated microglia derive solely from the local proliferation of resident microglia [64]. Aged mice can restore their aged microglial cell densities and morphologies (exhibiting similar phenotypes as young microglia) following the proliferation of surviving microglia after PLX5622mediated microglial depletion [71]. The newly repopulated microglia were beneficial in rescuing aged-related memory and long-term potentiation deficits in mice [71]. In support of this, a recent study demonstrated that repopulating microglia after experimental depletion using both genetic and pharmacological methods exhibit a pro-regenerative phenotype, which stimulated functional neurogenesis, improved spatial learning and memory deficits and promoted brain repair [79].

However, microglial depletion and repopulation by targeting either CSF1R or CX3CR1 may also affect CNS macrophages in the meninges or choroid, as well as other immune cells in the periphery [35, 80, 81]. In order to avoid potential effects on non-CNS resident macrophages, diphtheria toxin can be directly injected into the hippocampus to achieve selective microglia depletion in CX3CR $1{ }^{\text {CreERT2 }}$ iDTR mice. In concordance with previous results, the appearance of repopulating microglia in the hippocampus contributes to improve the behavioral functions [79]. In order to avoid potential effects from the periphery, PLX3397 was administrated in primary organotypic hippocampal slice culture to specifically target microglia without involvement of peripheral myeloid cells [82]. Consistent with previous results, the newly repopulated microglia exhibited anti-inflammatory properties by up-regulating the expression of IL-10, CX3CL1 and BDNF [82]. Microglial repopulation can also downregulate proinflammatory responses following LPS stimulation and persistent proinflammatory responses in the setting of chronic ethanol binging [82].

Different strategies for microglial replacement in the CNS or selective brain regions have been recently proposed [83]. Considering the beneficial effects of repopulating microglia in the CNS, it is tempting to hypothesize that replacing microglia in CSF1R-related leukoencephalopathy through a pharmacological depletion and repopulation paradigm may prolong the survival of patients, despite the newly repopulating microglia still carrying the CSF1R gene mutation. In preclinical studies, depletion of microglia can ameliorate cerebral white matter injury through reducing proinflammatory mediators [84]. Depleting microglia can also attenuate demyelination and neural damage in the mouse model carrying human PLP1 mutations, resembling progressive MS [85].

We propose that a combination of microglial replacement by the proliferation of resident microglia after depletion and other anti-inflammatory treatments that modulate the microenvironment may be more effective for CSF1R-related leukoencephalopathy. As a novel and still developing field, potential safety concerns during microglial depletion and repopulation remain a challenge. In preclinical studies microglia depletion does not show obvious side-effects [78], while depleting prenatal microglia may lead to long-term behavioral abnormalities [86]. An open-label study in patients with amyotrophic 
lateral sclerosis in order to characterize the safety, tolerability and microglial response following pharmacological CSF1R inhibition with BLZ945 is still ongoing in Stockholm (ClinicalTrials.gov, identifier: NCT04066244). Although the primary endpoint was not reached in a phase II clinical trial, microglial depletion using PLX3397 with an oral dose 600 or $1000 \mathrm{mg} / \mathrm{kg}$ was well-tolerated in patients with recurrent glioblastoma, and was evident in tumor tissues $[87,88]$. Some potential adverse events such as febrile neutropenia, drug-induced liver injury, anemia and hypertension were reported in some individuals [87]. It is also important to note that circulating CD $14{ }^{\text {dim }}{ }^{2} D 16^{+}$monocytes (non-classical monocytes) are significantly reduced after PLX3397 treatment [87, 88 ]. Since the numbers of non-classical monocytes can also be decreased with disease progression in CSF1Rrelated leukoencephalopathy patients [33], the clinical translation of pharmacological CSF1R inhibitors should be considered with caution.

\section{Microglial replacement by infiltrating microglia-like cells}

Apart from cell replacement by the proliferation of remaining host microglia following depletion [64], the engraftment of parenchymal microglia-like cells can be achieved under specific experimental circumstances once the microglial niche is made available $[44,65,66]$. We have previously demonstrated that microglia can be efficiently depleted ( $\sim 95 \%)$ by the administration of tamoxifen in $C X 3 C R 1^{\text {CreER/+ }}$ Rosa $26^{D T A /+}$ mice, causing intracellular DTA expression upon Cre recombination [44]. One month later the newly repopulated microglia in the CNS of these mice exhibited two distinct cell subpopulations differing in their expression of F4/80 [44]. Specifically, host microglia in the brain had a low expression of F4/80, while peripherally derived microglia-like cells in the brain expressed high levels of F4/80 and were P2ry12 $2^{-}[44]$. Along this line, a wave of Ly6C ${ }^{\text {hi }}$ monocytes were recruited into the brain of $\mathrm{C} x 3 \mathrm{cr} 1^{\mathrm{CreER}}$ ${ }^{1+}$ Rosa $26^{D T A /+}$ mice only 2 days post- tamoxifen injection and before the appearance of $\mathrm{F} 4 / 80^{\mathrm{hi}}$ microglia-like cells in the brain [44]. Furthermore, intravenous adoptive transfer of cultured Ly6C ${ }^{\text {hi }}$ monocytes in microglia-depleted recipient mice led to the reconstitution of $\mathrm{F} 4 / 80^{\mathrm{hi}}$ microglia-like cells with little contribution from the $\mathrm{F} 4 / 80^{\text {low }}$ (CNS-resident) microglial pool [44]. Our previous findings indicated that engrafted Ly $6 C^{\text {hi }}$ monocytes are shaped by the CNS microenvironment to gradually become microglial-like cells when the microglial niche is available after experimental ablation [54, 89], while remaining transcriptionally, epigenetically and functionally distinct from yolk-sac-derived resident microglia [44].
These findings are further supported by a recent study that convincingly demonstrated that graft-derived microglia-like cells can seed the brain parenchyma following irradiation, a condition that makes the microglial niche available [66]. Through local clonal expansion the monocyte-derived microglia-like cells competed with tissue resident microglia for space and expressed genes such as Ccr2, Apoe, Ifnar1 and Ms4a7 [66]. As host-resident microglia and engrafted microglia-like cells yield distinct responses to LPS challenge [66], it is still important to question how a microglia-like cell repopulated CNS will function physiologically.

Basic research indicates that transplanted bone marrow-derived cells can rescue the phenotypes of Csf1rnull mice [89]. Bone marrow-derived microglia-like cells have a greater ability to clear cellular debris and dead cells compared to resident microglia, depending on different disease conditions [66]. Bone marrow-derived cells stimulated with CSF-1 upregulated microglial-specific surface markers such as Tmem119, and increased their functional ability to phagocytose amyloid- $\beta$ peptides in vitro [90]. Hippocampal transplantation of bone marrow-derived microglia-like cells in an Alzheimer's mouse model increased the phagocytic uptake of amyloid- $\beta$, leading to reduced amyloid- $\beta$ burden in the brain and improved cognitive deficits [90]. Bone marrow-derived cells stimulated with granulocyte CSF (G-CSF) home and reside in the brain after radiation injury, modulating functional repair and restoring white matter damage [91].

Microglial replacement by infiltrating microglia-like cells thus provides an attractive possibility to remove dysfunctional microglia in patients with CSF1R-related leukoencephalopathy [25]. Furthermore, targeting the CX3CL1-CX3CR1 signaling pathway regulates the process of microglial repopulation from the proliferation of resident microglia in the CNS [92]. A combination of repopulation of microglia after depletion and bone marrow transplantation may serve as a novel therapeutic platform for CSF1R-related leukoencephalopathy. Clinically, the derivation of microglia-like cells from healthy donor circulating monocytes could be another option for replacing dysfunctional microglia (Fig. 2) [93]. However, the precise role of these engrafted microglia-like cells in the brain needs further investigation [94].

\section{Microglial replacement by hematopoietic stem cell transplantation}

Empiric trials of glucocorticoids did not show obvious clinical improvements in reported CSF1R-related leukoencephalopathy cases [95]. Hematopoietic stem cell transplantation (HSCT) offers a potential regenerative strategy for patients in order to halt disease progression and improve survival (Fig. 2). A previous study reported 


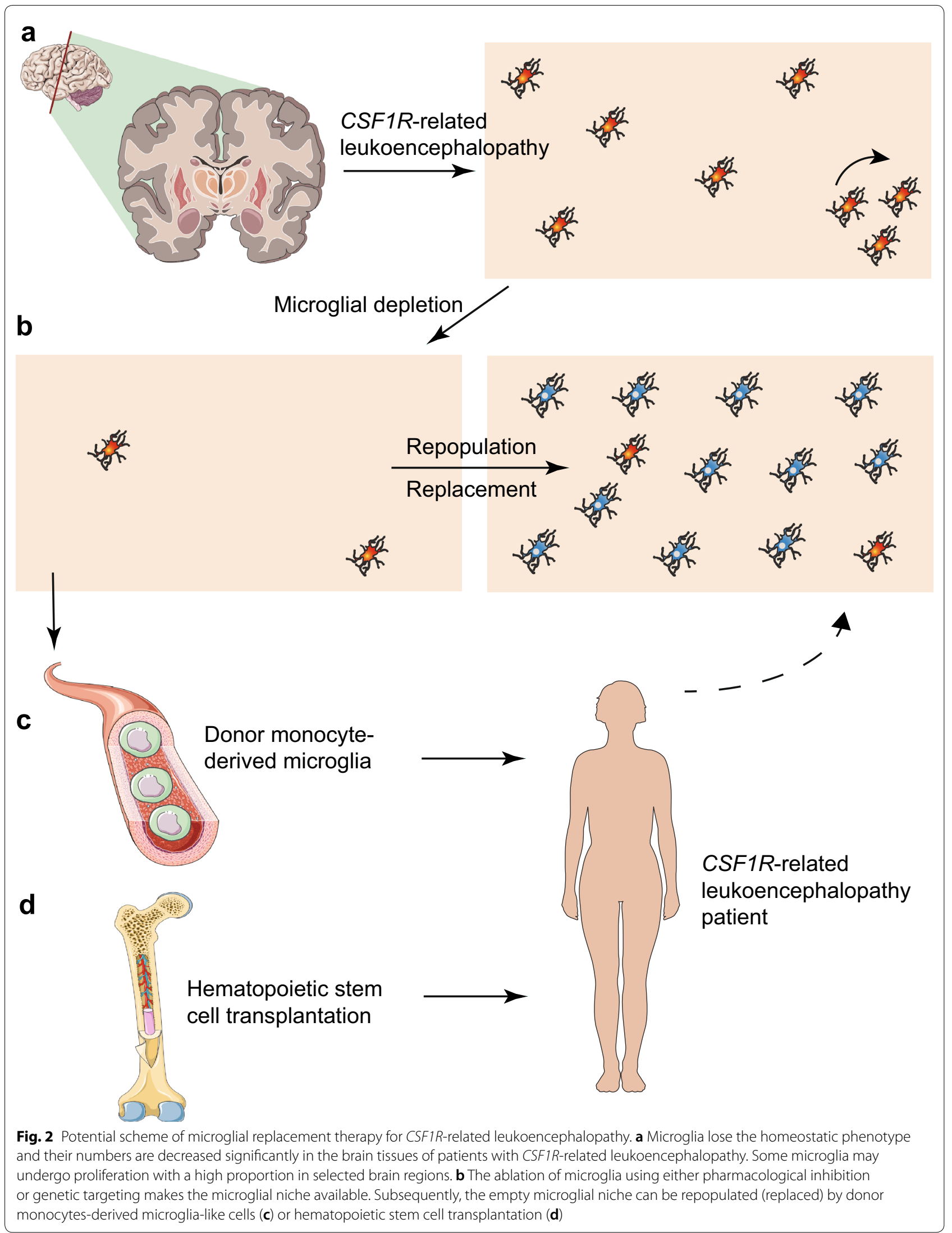


an affected individual who underwent HSCT from her sibling remained stable for more than 15 years [96]. This meaningful finding is further supported by a recent study convincingly showing the effectiveness of HSCT from human leukocyte antigen-matched wild-type CSF1R gene donors in two CSF1R-related leukoencephalopathy patients [95]. These patients did not show any signs of acute or chronic graft-versus-host disease after HSCT, but experienced worsened neurological symptoms such as parkinsonism after transplantation [95]. During the follow-up period one patient developed pneumonia and the other suffered from new localization-related seizures. Reducing intensity-conditioning regimens is required in order to minimize potential toxicity during HSCT [95]. More importantly, the progression of cognitive decline, overall clinical outcomes and gait impairment gradually stabilized in 9 and 6 years post-HSCT for each patient [95]. Furthermore, in brain MRIs the T2 lesions and fluid-attenuated inversion recovery abnormalities were gradually reduced, and there was a significant resolution of abnormal multifocal reduced diffusion as measured by MRI after 2 years [95]. In both cases peripheral blood donor chimerism was successfully established after transplantation but the degree of chimerism in the CNS is not known since the patients are still alive [95]. Satisfactory long-term effects of HSCT on CSF1R-related leukoencephalopathy were noted in another case [97]. This patient also experienced deteriorated pyramidal symptoms 3 months after HSCT, while gradually improved during long-term follow-up [97].

Graft-derived ramified $\mathrm{Iba}^{+}$cells from a sex-mismatched donor was noted in post-mortem brains after HSCT through observation of Y-chromosomes using chromogenic in situ hybridization [66]. In an experimental model of demyelination, intraventricular transplantation of mesenchymal stem cells exerted neuroprotective effects by upregulating the expression of the CX3CL1/ CX3CR1 axis, shifting proinflammatory microglia into an anti-inflammatory state and resulting in remyelination [98]. These labeled mesenchymal stem cells were present in the corpus callosum 2 weeks after transplantation [98]. In addition to their neurotrophic support and immunomodulation, stem cell grafts may also exercise beneficial effects through structural cell replacement [99]. The function of phagocytosis can also be modulated by grafted stem cells in animal models [99]. Transplantation of mesenchymal stem cells can promote disease recovery in an intraventricular hemorrhage rat model mainly by blocking reactive microglia, modulating the phosphorylation of STAT1 and p38 MAPK, and consequently reducing the production of proinflammatory cytokines [100].

All major brain cell types including microglia can be generated and differentiated from stem cells [101].
Although microglial replacement by HSCT is far from perfect, allogeneic HSCT could be beneficial in patients with CSF1R-related leukoencephalopathy in clinical practice by providing brain-engrafting microglia-like cells with donor wild-type CSF1R to repopulate the microglial niche [2]. However, to what extent HSCT can replace the resident microglia remains unclear.

Repopulating microglia from a human leukocyte antigen-matched donor could perform normal functions such as phagocytosis and cytokine production, while altered functions such as an increased release of cytokines after stimulation were noted in microglialike cells derived from either a patient donor or a family member who also carried the CSF1R gene mutation [101]. Worsening clinical symptoms post-HSCT may be related to replacing microglia and phagocytosing debris in the brain. Finally, partial clinical stabilization and resolution of MRI abnormalities several years after HSCT may result from the replacement of some dysfunctional microglia by new microglia that are maintained in the CNS. It is also important to note that some early-onset CSF1R-related leukoencephalopathy patients did not show clinical symptom improvement following HSCT [102], suggesting that selection of the right patients, adequate design of clinical trials for rare diseases and long-term clinical monitoring with standardized, internationally validated tools will be necessary for evaluation of clinical outcomes and experimental treatment endpoints. Tissue resident macrophages in the CNS can be generated from stem cells, while only partially resembling embryonic-derived resident microglia [103]. Recreating the microglial niche and complex niche factors such as sex differences and cell-to cell communications should be considered when designing potential microglia replacement therapy.

\section{Concluding remarks}

There are no available specific therapies for CSF1Rrelated leukoencephalopathy to date, and development of a potential microglia-based treatment is warranted. A detailed understanding of the microglial niche, and potential regulating factors such as epigenetic markers, novel pharmacological therapies to remove microglia by targeting CSF1R, emerging genomic engineering tools and stem cell treatments, may all facilitate the selective replacement of dysfunctional microglia with healthy microglia in CSF1R-related leukoencephalopathy patients. While still relatively novel, microglial replacement therapy is a scientifically sound strategy and presents an exciting research opportunity that could lead to clinical translation, alleviation of symptoms and improving the life quality of individuals who are suffering from currently incurable CSF1R-related leukoencephalopathy. 


\begin{abstract}
Abbreviations
AARS: Alanyl tRNA synthetase; AD: Alzheimer's disease; BDNF: Brain-derived neurotrophic factor; CADASIL: Cerebral autosomal dominant arteriopathy with subcortical infarcts and leukoencephalopathy; CNS: Central nervous system; CSF1R: Colony stimulating factor 1 receptor; HDLS: Hereditary diffuse leukoencephalopathy with spheroids; HSCT: Hematopoietic stem cell transplantation; G-CSF: Granulocyte CSF; LPS: Lipopolysaccharide; MRI: Magnetic resonance imaging; MS: Multiple sclerosis; POLD: Pigmented orthochromatic leukodystrophy.
\end{abstract}

\section{Acknowledgements}

We would like to thank Tobias Granberg from the Department of Radiology at Karolinska University Hospital and Daniel F. Broderick from the Department of Radiology at Mayo Clinic Florida for their help regarding MRI features of CSF1Rrelated leukoencephalopathy.

\section{Authors' contributions}

$\mathrm{JH}$ and RAH generated the outline of the Review. JH wrote the first draft. JH, HS and ZKW prepared the figures. HS, ZKW, VDK and RAH revised the manuscript and approved the final version. All authors read and approved the final manuscript.

\section{Funding}

Open Access funding provided by Karolinska Institute. This work was supported by grants from the China Scholarship Council, the Swedish Medical Research Council, Neuroforbundet, Alltid Lite Sterkere, HjärnFonden, AlzheimerFonden and BarnCancerFonden. ZKW is partially supported by the Mayo Clinic Center for Regenerative Medicine, the gifts from The Sol Goldman Charitable Trust, and the Donald G. and Jodi P. Heeringa Family, the Haworth Family Professorship in Neurodegenerative Diseases Fund, and The Albertson Parkinson's Research Foundation. VDK has received financial support from Stockholm County Council (Grant ALF 20160457), Biogen (recipient of grant and scholarship, PI for the project sponsored by Biogen); Novartis (Scientific Advisory Board member, recipient of scholarship and lecture honoraria) and Merck (Scientific Advisory Board member, recipient of lecture honoraria).

\section{Availability of data and materials}

$$
\text { Not applicable. }
$$

\section{Ethics approval and consent to participate}

Not applicable.

\section{Consent for publication}

Not applicable.

\section{Competing interests}

ZKW serves as PI or Co-PI on Biogen, Inc. (228PD201), Biohaven Pharmaceuticals, Inc. (BHV4157-206 and BHV3241-301), and Neuraly, Inc. (NLY01-PD-1) grants. He serves as Co-PI of the Mayo Clinic APDA Center for Advanced Research.

\section{Author details}

${ }^{1}$ Applied Immunology and Immunotherapy, Department of Clinical Neuroscience, Karolinska Institutet, Center for Molecular Medicine, Karolinska University Hospital, Solna, Sweden. ${ }^{2}$ Department of Neurology, Mayo Clinic, Jacksonville, USA. ${ }^{3}$ Department of Clinical Neuroscience, Karolinska Institutet, Stockholm, Sweden. ${ }^{4}$ Department of Neurology, Karolinska University Hospital, Stockholm, Sweden.

Received: 17 October 2020 Accepted: 23 November 2020

Published online: 07 December 2020

\section{References}

1. Wider C, Wszolek ZK (2014) Hereditary diffuse leukoencephalopathy with axonal spheroids: more than just a rare disease. Neurology 82(2):102-103

2. Konno T, Kasanuki K, Ikeuchi T, Dickson DW, Wszolek ZK (2018) CSF1Rrelated leukoencephalopathy: a major player in primary microgliopathies. Neurology 91(24):1092-1104
3. Konno T, Yoshida K, Mizuta I et al (2018) Diagnostic criteria for adultonset leukoencephalopathy with axonal spheroids and pigmented glia due to CSF1R mutation. Eur J Neurol 25(1):142-147

4. Adams SJ, Kirk A, Auer RN (2018) Adult-onset leukoencephalopathy with axonal spheroids and pigmented glia (ALSP): integrating the literature on hereditary diffuse leukoencephalopathy with spheroids (HDLS) and pigmentary orthochromatic leukodystrophy (POLD). J Clin Neurosci 48:42-49

5. Axelsson R, Roytta M, Sourander P, Akesson HO, Andersen O (1984) Hereditary diffuse leucoencephalopathy with spheroids. Acta Psychiatr Scand Suppl 314:1-65

6. Sundal C, Carmona S, Yhr M et al (2019) An AARS variant as the likely cause of Swedish type hereditary diffuse leukoencephalopathy with spheroids. Acta Neuropathol Commun 7(1):188

7. Nicholson AM, Baker MC, Finch NA et al (2013) CSF1R mutations link POLD and HDLS as a single disease entity. Neurology 80(11):1033-1040

8. Konno T, Yoshida K, Mizuno T et al (2017) Clinical and genetic characterization of adult-onset leukoencephalopathy with axonal spheroids and pigmented glia associated with CSF1R mutation. Eur J Neurol 24(1):37-45

9. Sundal C, Baker M, Karrenbauer V et al (2015) Hereditary diffuse leukoencephalopathy with spheroids with phenotype of primary progressive multiple sclerosis. Eur J Neurol 22(2):328-333

10. Sharma R, Graff-Radford J, Rademakers R, Boeve BF, Petersen RC, Jones DT (2019) CSF1R mutation presenting as dementia with Lewy bodies. Neurocase 25(1-2):17-20

11. Zhuang LP, Liu CY, Li YX, Huang HP, Zou ZY (2020) Clinical features and genetic characteristics of hereditary diffuse leukoencephalopathy with spheroids due to CSF1R mutation: a case report and literature review. Ann Transl Med 8(1):11

12. Sundal C, Van Gerpen JA, Nicholson AM et al (2012) MRI characteristics and scoring in HDLS due to CSF1R gene mutations. Neurology 79(6):566-574

13. Baba Y, Ghetti B, Baker MC et al (2006) Hereditary diffuse leukoencephalopathy with spheroids: clinical, pathologic and genetic studies of a new kindred. Acta Neuropathol 111(4):300-311

14. Li S, Zhu Y, Yao M (2020) Spinal cord involvement in adult-onset leukoencephalopathy with axonal spheroids and pigmented glia. JAMA Neurol 77:1169-1170

15. Shu Y, Long L, Liao S et al (2016) Involvement of the optic nerve in mutated CSF1R-induced hereditary diffuse leukoencephalopathy with axonal spheroids. BMC Neurol 16:171

16. Konno T, Broderick DF, Mezaki N et al (2017) Diagnostic value of brain calcifications in adult-onset leukoencephalopathy with axonal spheroids and pigmented glia. AJNR Am J Neuroradiol 38(1):77-83

17. Mao C, Zhou L, Zhou L et al (2020) Biopsy histopathology in the diagnosis of adult-onset leukoencephalopathy with axonal spheroids and pigmented glia (ALSP). Neurol Sci 41(2):403-409

18. Granberg T, Hashim F, Andersen O, Sundal C, Karrenbauer VD (2016) Hereditary diffuse leukoencephalopathy with spheroids-a volumetric and radiological comparison with multiple sclerosis patients and healthy controls. Eur J Neurol 23(4):817-822

19. Mangeat G, Ouellette R, Wabartha M et al (2020) Machine learning and multiparametric brain MRI to differentiate hereditary diffuse leukodystrophy with spheroids from multiple sclerosis. J Neuroimaging 30:674-682

20. Khalil M, Teunissen CE, Otto M et al (2018) Neurofilaments as biomarkers in neurological disorders. Nat Rev Neurol 14(10):577-589

21. Hayer SN, Krey I, Barro C et al (2018) NfL is a biomarker for adult-onset leukoencephalopathy with axonal spheroids and pigmented glia. Neurology 91(16):755-757

22. Shi T, Li J, Tan C, Chen J (2019) Diagnosis of hereditary diffuse leukoencephalopathy with neuroaxonal spheroids based on next-generation sequencing in a family: case report and literature review. Medicine (Baltimore) 98(22):e15802

23. Horti AG, Naik R, Foss CA et al (2019) PET imaging of microglia by targeting macrophage colony-stimulating factor 1 receptor (CSF1R). Proc Natl Acad Sci USA 116(5):1686-1691

24. Han J, Harris RA, Zhang XM (2017) An updated assessment of microglia depletion: current concepts and future directions. Mol Brain 10(1):25 
25. Han J, Zhu K, Zhang XM, Harris RA (2019) Enforced microglial depletion and repopulation as a promising strategy for the treatment of neurological disorders. Glia 67(2):217-231

26. Chitu V, Gokhan S, Gulinello M et al (2015) Phenotypic characterization of a CSF1R haploinsufficient mouse model of adult-onset leukodystrophy with axonal spheroids and pigmented glia (ALSP). Neurobiol Dis $74: 219-228$

27. Oosterhof N, Chang IJ, Karimiani EG et al (2019) Homozygous mutations in CSF1R cause a pediatric-onset leukoencephalopathy and can result in congenital absence of microglia. Am J Hum Genet 104(5):936-947

28. Guo L, Bertola DR, Takanohashi A et al (2019) Bi-allelic CSF1R mutations cause skeletal dysplasia of dysosteosclerosis-pyle disease spectrum and degenerative encephalopathy with brain malformation. Am J Hum Genet 104(5):925-935

29. Kana V, Desland FA, Casanova-Acebes M et al (2019) CSF-1 controls cerebellar microglia and is required for motor function and social interaction. J Exp Med 216(10):2265-2281

30. Yang $X$, Huang $P$, Tan Y, Xiao Q (2019) A novel splicing mutation in the CSF1R gene in a family with hereditary diffuse leukoencephalopathy with axonal spheroids. Front Genet 10:491

31. Kim SI, Jeon B, Bae J et al (2019) An autopsy proven case of CSF1Rmutant adult-onset leukoencephalopathy with axonal spheroids and pigmented glia (ALSP) with premature ovarian failure. Exp Neurobiol 28(1):119-129

32. Cohen AS, Wilson SL, Trinh J, Ye XC (2015) Detecting somatic mosaicism: considerations and clinical implications. Clin Genet 87(6):554-562

33. Kraya T, Quandt D, Pfirrmann T et al (2019) Functional characterization of a novel CSF1R mutation causing hereditary diffuse leukoencephalopathy with spheroids. Mol Genet Genomic Med 7(4):e00595

34. Irvine KM, Caruso M, Cestari MF et al (2020) Analysis of the impact of CSF-1 administration in adult rats using a novel Csf1 r-mApple reporter gene. J Leukoc Biol 107(2):221-235

35. Lei F, Cui N, Zhou C, Chodosh J, Vavvas DG, Paschalis El (2020) CSF1R inhibition by a small-molecule inhibitor is not microglia specific; affecting hematopoiesis and the function of macrophages. Proc Natl Acad Sci USA 117:23336-23338

36. Hume DA, Caruso M, Ferrari-Cestari M, Summers KM, Pridans C, Irvine KM (2020) Phenotypic impacts of CSF1R deficiencies in humans and model organisms. J Leukoc Biol 107(2):205-219

37. Luo J, Elwood F, Britschgi M et al (2013) Colony-stimulating factor 1 receptor (CSF1R) signaling in injured neurons facilitates protection and survival. J Exp Med 210(1):157-172

38. Pridans C, Raper A, Davis GM et al (2018) Pleiotropic impacts of macrophage and microglial deficiency on development in rats with targeted mutation of the CSF1R locus. J Immunol 201 (9):2683-2699

39. Hanamsagar R, Bilbo SD (2017) Environment matters: microglia function and dysfunction in a changing world. Curr Opin Neurobiol 47:146-155

40. Kempthorne L, Yoon H, Madore C et al (2020) Loss of homeostatic microglial phenotype in CSF1R-related leukoencephalopathy. Acta Neuropathol Commun 8(1):72

41. Tada M, Konno T, Tada M et al (2016) Characteristic microglial features in patients with hereditary diffuse leukoencephalopathy with spheroids. Ann Neurol 80(4):554-565

42. Oosterhof N, Kuil LE, van der Linde HC et al (2018) Colony-stimulating factor 1 receptor (CSF1R) regulates microglia density and distribution, but not microglia differentiation in vivo. Cell Rep 24(5):1203-1217

43. Elmore MR, Najafi AR, Koike MA et al (2014) Colony-stimulating factor 1 receptor signaling is necessary for microglia viability, unmasking a microglia progenitor cell in the adult brain. Neuron 82(2):380-397

44. Lund H, Pieber M, Parsa R et al (2018) Competitive repopulation of an empty microglial niche yields functionally distinct subsets of microglialike cells. Nat Commun 9(1):4845

45. Tian WT, Zhan FX, Liu Q et al (2019) Clinicopathologic characterization and abnormal autophagy of CSF1R-related leukoencephalopathy. Transl Neurodegener 8:32

46. Hanamsagar R, Bilbo SD (2016) Sex differences in neurodevelopmental and neurodegenerative disorders: focus on microglial function and neuroinflammation during development. J Steroid Biochem Mol Biol 160:127-133
47. Guneykaya D, Ivanov A, Hernandez DP et al (2018) Transcriptional and translational differences of microglia from male and female brains. Cell Rep 24(10):2773-2783

48. Villa A, Gelosa P, Castiglioni L et al (2018) Sex-specific features of microglia from adult mice. Cell Rep 23(12):3501-3511

49. Kodama L, Gan L (2019) Do microglial sex differences contribute to sex differences in neurodegenerative diseases? Trends Mol Med 25(9):741-749

50. Murtaj V, Belloli S, Di Grigoli G et al (2019) Age and sex influence the neuro-inflammatory response to a peripheral acute LPS challenge. Front Aging Neurosci 11:299

51. Rossetti AC, Paladini MS, Trepci A et al (2019) Differential neuroinflammatory response in male and female mice: a role for BDNF. Front Mol Neurosci 12:166

52. Thion MS, Low D, Silvin A et al (2018) Microbiome influences prenatal and adult microglia in a sex-specific manner. Cell 172(3):500-516

53. Han J, Zhu K, Zhou K et al (2020) Sex-specific effects of microglia-like cell engraftment during experimental autoimmune encephalomyelitis. Int J Mol Sci 21(18):1-16

54. Guilliams M, Scott CL (2017) Does niche competition determine the origin of tissue-resident macrophages? Nat Rev Immunol 17(7):451-460

55. Guilliams M, Thierry GR, Bonnardel J, Bajenoff M (2020) Establishment and maintenance of the macrophage niche. Immunity 52(3):434-451

56. Ginhoux F, Guilliams M (2016) Tissue-resident macrophage ontogeny and homeostasis. Immunity 44(3):439-449

57. Askew K, Li K, Olmos-Alonso A et al (2017) Coupled proliferation and apoptosis maintain the rapid turnover of microglia in the adult brain. Cell Rep 18(2):391-405

58. Scott CL, Zheng F, De Baetselier P et al (2016) Bone marrow-derived monocytes give rise to self-renewing and fully differentiated Kupffer cells. Nat Commun 7:10321

59. van de Laar L, Saelens W, De Prijck S et al (2016) Yolk sac macrophages, fetal liver, and adult monocytes can colonize an empty niche and develop into functional tissue-resident macrophages. Immunity 44(4):755-768

60. Ginhoux F, Greter M, Leboeuf M et al (2010) Fate mapping analysis reveals that adult microglia derive from primitive macrophages. Science 330(6005):841-845

61. Tsuji S, Di Martino E, Mukai T et al (2020) Aggravated brain injury after neonatal hypoxic ischemia in microglia-depleted mice. J Neuroinflammation 17(1):111

62. Rice RA, Pham J, Lee RJ, Najafi AR, West BL, Green KN (2017) Microglial repopulation resolves inflammation and promotes brain recovery after injury. Glia 65(6):931-944

63. Najafi AR, Crapser J, Jiang S et al (2018) A limited capacity for microglial repopulation in the adult brain. Glia 66(11):2385-2396

64. Huang Y, Xu Z, Xiong S et al (2018) Repopulated microglia are solely derived from the proliferation of residual microglia after acute depletion. Nat Neurosci 21(4):530-540

65. Cronk JC, Filiano AJ, Louveau A et al (2018) Peripherally derived macrophages can engraft the brain independent of irradiation and maintain an identity distinct from microglia. J Exp Med 215(6):1627-1647

66. Shemer A, Grozovski J, Tay TL et al (2018) Engrafted parenchymal brain macrophages differ from microglia in transcriptome, chromatin landscape and response to challenge. Nat Commun 9(1):5206

67. Zhu K, Pieber M, Han J et al (2020) Absence of microglia or presence of peripherally-derived macrophages does not affect tau pathology in young or old hTau mice. Glia 68(7):1466-1478

68. Parkhurst CN, Yang G, Ninan I et al (2013) Microglia promote learningdependent synapse formation through brain-derived neurotrophic factor. Cell 155(7):1596-1609

69. Ferrini F, De Koninck Y (2013) Microglia control neuronal network excitability via BDNF signalling. Neural Plast 2013:429815

70. Bosurgi L, Hughes LD, Rothlin CV, Ghosh S (2017) Death begets a new beginning. Immunol Rev 280(1):8-25

71. Elmore MRP, Hohsfield LA, Kramar EA et al (2018) Replacement of microglia in the aged brain reverses cognitive, synaptic, and neuronal deficits in mice. Aging Cell 17(6):e12832

72. Zhang LY, Pan J, Mamtilahun M et al (2020) Microglia exacerbate white matter injury via complement C3/C3aR pathway after hypoperfusion. Theranostics 10(1):74-90 
73. Liu Y, Given KS, Dickson EL, Owens GP, Macklin WB, Bennett JL (2019) Concentration-dependent effects of CSF1R inhibitors on oligodendrocyte progenitor cells ex vivo and in vivo. Exp Neurol 318:32-41

74. Beckmann N, Giorgetti E, Neuhaus A et al (2018) Brain region-specific enhancement of remyelination and prevention of demyelination by the CSF1R kinase inhibitor BLZ945. Acta Neuropathol Commun 6(1):9

75. Gerber YN, Saint-Martin GP, Bringuier CM et al (2018) CSF1R inhibition reduces microglia proliferation, promotes tissue preservation and improves motor recovery after spinal cord injury. Front Cell Neurosci $12: 368$

76. Du X, Xu Y, Chen S, Fang M (2020) Inhibited CSF1R alleviates ischemia injury via inhibition of microglia M1 polarization and NLRP3 pathway. Neural Plast 2020:8825954

77. Mancuso R, Fryatt G, Cleal M et al (2019) CSF1R inhibitor JNJ-40346527 attenuates microglial proliferation and neurodegeneration in P301S mice. Brain 142(10):3243-3264

78. Tanabe S, Saitoh S, Miyajima H, Itokazu T, Yamashita T (2019) Microglia suppress the secondary progression of autoimmune encephalomyelitis. Glia 67(9):1694-1704

79. Willis EF, MacDonald KPA, Nguyen QH et al (2020) Repopulating microglia promote brain repair in an IL-6-dependent manner. Cell 180(5):833-846

80. Shi Y, Manis M, Long J et al (2019) Microglia drive APOE-dependent neurodegeneration in a tauopathy mouse model. J Exp Med 216(11):2546-2561

81. Han J, Fan Y, Zhou K et al (2020) Underestimated peripheral effects following pharmacological and conditional genetic microglial depletion. Int J Mol Sci 21(22):8603

82. Coleman LG Jr, Zou J, Crews FT (2020) Microglial depletion and repopulation in brain slice culture normalizes sensitized proinflammatory signaling. J Neuroinflamm 17(1):27

83. Xu Z, Rao Y, Huang $Y$ et al (2020) Efficient strategies for microglia replacement in the central nervous system. Cell Rep 32(6):10804

84. Kakae M, Tobori S, Morishima M, Nagayasu K, Shirakawa H, Kaneko S (2019) Depletion of microglia ameliorates white matter injury and cognitive impairment in a mouse chronic cerebral hypoperfusion model. Biochem Biophys Res Commun 514(4):1040-1044

85. Groh J, Klein D, Berve K, West BL, Martini R (2019) Targeting microglia attenuates neuroinflammation-related neural damage in mice carrying human PLP1 mutations. Glia 67(2):277-290

86. Nelson LH, Lenz KM (2017) Microglia depletion in early life programs persistent changes in social, mood-related, and locomotor behavior in male and female rats. Behav Brain Res 316:279-293

87. Wesolowski R, Sharma N, Reebel L et al (2019) Phase lb study of the combination of pexidartinib (PLX3397), a CSF-1R inhibitor, and paclitaxel in patients with advanced solid tumors. Ther Adv Med Oncol 11:1758835919854238

88. Butowski N, Colman H, De Groot JF et al (2016) Orally administered colony stimulating factor 1 receptor inhibitor $\mathrm{PLX} 3397$ in recurrent glioblastoma: an Ivy Foundation Early Phase Clinical Trials Consortium phase II study. Neuro Oncol 18(4):557-564

89. Bennett FC, Bennett ML, Yaqoob F et al (2018) A combination of ontogeny and CNS environment establishes microglial identity. Neuron 98(6):1170-1183
90. Kawanishi S, Takata K, Itezono S et al (2018) Bone-marrow-derived microglia-like cells ameliorate brain amyloid pathology and cognitive impairment in a mouse model of Alzheimer's disease. J Alzheimers Dis 64(2):563-585

91. Dietrich J, Baryawno N, Nayyar N et al (2018) Bone marrow drives central nervous system regeneration after radiation injury. J Clin Investig 128(1):281-293

92. Zhang $Y$, Zhao L, Wang $X$ et al (2018) Repopulating retinal microglia restore endogenous organization and function under CX3CL1-CX3CR1 regulation. Sci Adv 4(3):eaap8492

93. Sellgren CM, Sheridan SD, Gracias J, Xuan D, Fu T, Perlis RH (2017) Patient-specific models of microglia-mediated engulfment of synapses and neural progenitors. Mol Psychiatry 22(2):170-177

94. Hohsfield LA, Najafi AR, Ghorbanian Y et al (2020) Effects of long-term and brain-wide colonization of peripheral bone marrow-derived myeloid cells in the CNS. J Neuroinflammation 17(1):279

95. Gelfand JM, Greenfield AL, Barkovich M et al (2020) Allogeneic HSCT for adult-onset leukoencephalopathy with spheroids and pigmented glia. Brain 143(2):503-511

96. Eichler FS, Li J, Guo Y et al (2016) CSF1R mosaicism in a family with hereditary diffuse leukoencephalopathy with spheroids. Brain 139(Pt 6):1666-1672

97. Mochel F, Delorme C, Czernecki V et al (2019) Haematopoietic stem cell transplantation in CSF1R-related adult-onset leukoencephalopathy with axonal spheroids and pigmented glia. J Neurol Neurosurg Psychiatry 90(12):1375-1376

98. Barati S, Ragerdi Kashani I, Moradi F et al (2019) Mesenchymal stem cell mediated effects on microglial phenotype in cuprizone-induced demyelination model. J Cell Biochem 120(8):13952-13964

99. Shen Z, Li X, Bao X, Wang R (2017) Microglia-targeted stem cell therapies for Alzheimer disease: a preclinical data review. J Neurosci Res 95(12):2420-2429

100. Kim S, Kim YE, Hong S et al (2020) Reactive microglia and astrocytes in neonatal intraventricular hemorrhage model are blocked by mesenchymal stem cells. Glia 68(1):178-192

101. Penney J, Ralvenius WT, Tsai LH (2020) Modeling Alzheimer's disease with iPSC-derived brain cells. Mol Psychiatry 25(1):148-167

102. Wang M, Zhang X (2019) A novel CSF-1R mutation in a family with hereditary diffuse leukoencephalopathy with axonal spheroids misdiagnosed as hydrocephalus. Neurogenetics 20(3):155-160

103. Claes C, Van den Daele J, Verfaillie CM (2018) Generating tissue-resident macrophages from pluripotent stem cells: lessons learned from microglia. Cell Immunol 330:60-67

\section{Publisher's Note}

Springer Nature remains neutral with regard to jurisdictional claims in published maps and institutional affiliations.

Ready to submit your research? Choose BMC and benefit from

- fast, convenient online submission

- thorough peer review by experienced researchers in your field

- rapid publication on acceptance

- support for research data, including large and complex data types

- gold Open Access which fosters wider collaboration and increased citations

- maximum visibility for your research: over 100M website views per year

At BMC, research is always in progress.

Learn more biomedcentral.com/submissions 